\title{
Flu-like symptoms and hyperechoic foetal bowel
}

\author{
N Abusheikha, O Sallha, S Marcus, M Johnstone
}

A 26-year-old woman attended the antenatal clinic at 12 weeks gestation in her first pregnancy having conceived spontaneously following 4 years of unexplained subfertility. An ultrasound scan confirmed the presence of a singleton, viable intra-uterine pregnancy with a crown-rump length equivalent to 12 weeks. Routine investigations revealed that her blood group was Rhesus positive, she was rubella immune and TORCH screen was negative. At 15 weeks gestation a triple test showed that her chances of having a Down's syndrome baby was $1: 250$. However, a foetal anomaly scan performed at 19 weeks demonstrated a region of hyperechoic bowel occupying the lower part of the foetal abdomen (figures). The scan showed no other structural defects and there were no ultrasonic markers for chromosomal anomalies. The couple were fully counselled and declined any invasive diagnostic procedures. A repeat scan at 24 weeks gestation confirmed the original findings and showed no change in the size of the abnormality. A week later the patient presented with loss of foetal movements. An ultrasound scan confirmed intra-uterine foetal death. Labour was induced with Gemeprost vaginal pessaries and after the fourth dose the patient delivered a 600 -g stillborn male infant and a large pale placenta. The patient declined submortum examination. Although she initially denied any systemic illness, on closer questioning, it transpired that she had had flu-like symptoms for few days at 10 weeks gestation which settled spontaneously.

\begin{abstract}
Aintree Centre for Reproductive Medicine, Fazakerly Hospital, Liverpool, UK

N Abusheikha

M Johnstone
\end{abstract}

\section{Assisted Conception Unit, St James \\ University Hospital, Leeds, UK}

O Sallha

Bourne Hall Clinic, Bourn,

Cambridgeshire, UK

S Marcus

Correspondence to

N Abusheikha, Bourne Hall

Clinic, Bourn,

Cambridgeshire CB3 7TR, UK

Accepted 13 May 1998

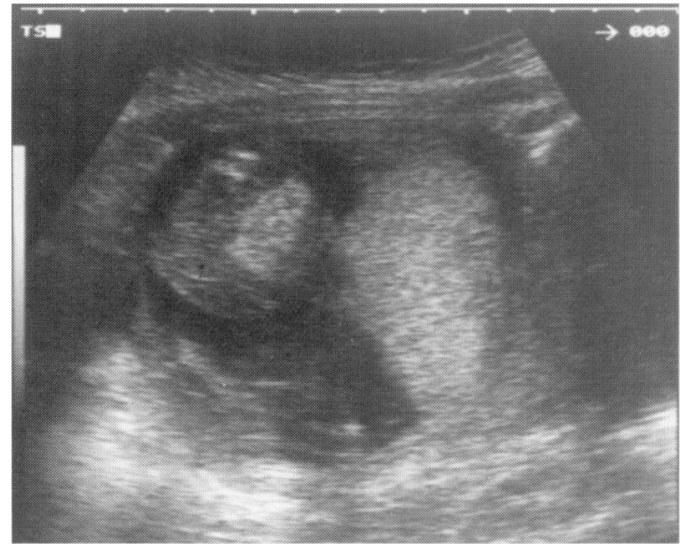

Figure 1 Ultrasound scan at 19 weeks gestation

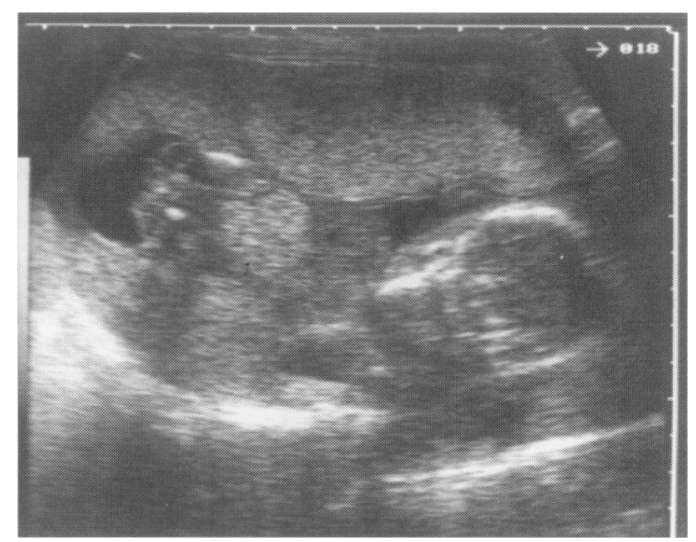

Figure 2 Ultrasound scan at 19 weeks gestation

\section{Questions}

1 What are the possible causes of the hyperechoic bowel ?

2 What investigations could be performed to aid the diagnosis ? 


\section{Answers}

QUESTION 1

The possible causes of a hyperechoic bowel are a normal variant, congenital cytomegalovirus (CMV) infection or Down's syndrome.

QUESTION 2

Maternal serum screening for recent CMV infection, and foetal karyotyping would aid diagnosis.

\section{Discussion}

Although hyperechoic bowel can be a normal variant, it has been reported in cases of both congenital CMV infection and Down's syndrome. ${ }^{1}$ Differentiation can be difficult due to the presence of other common markers. Peters et al reported a case of congenital CMV with hyperechoic foetal bowel, short biometry, and abnormal triple screen results. In this case maternal history of flu-like symptoms, normal triple screen results, and absence of other sonographic markers of Down's syndrome allowed us to consider the diagnosis of CMV infection, which was upheld by maternal seroconversion.

Prenatal diagnosis is feasible and reliable. ${ }^{3}$ The techniques that are available for foetal sampling include amniocentesis, chordocente- sis and chorionic villous sampling. Our patient declined any procedure that might carry any risk to her baby. Her decision may have been affected by her 4-year history of subfertility.

Congenital CMV disease occurs in about $30-40 \%$ of women with primary infection, and in less than $1 \%$ of immune women. ${ }^{4}$ Unfortunately no in-utero treatment has been shown to be effective. In the most severe cases, early termination of pregnancy is justified. ${ }^{5}$ Similarly, there are no current protocols for the use of antiviral agents during pregnancy, to decrease the risk of vertical transmission of CMV infection. A live attenuated CMV vaccine (Towne strain) is available and has been used in renal transplant patients. ${ }^{6}$ Possible benefits and risks of this vaccine for susceptible pregnant women have not been established. Very recent advances have established the synthesis of $\mathrm{CMV}$ envelope proteins which may be used for immunisation using recombinant DNA techniques. ${ }^{7}$ However, the use of this recombinant protein as a vaccine is still under investigation.

\section{Final diagnosis}

\section{Congenital CMV infection.}

Keywords: cytomegalovirus; seroconversion; hyperechoic bowel; prenatal diagnosis
1 Bahado-Singh R, Morotti R, Copel JA, Mohany MJ. Hyperechoic foetal bowel: the prenatal consequences. Prenat Diagn 1994;14:981-7.

2 Peters MT, Lowe TW, Carpenter A, Kole S. Prenatal diagnosis of cytomegalovirus infection with abnormal triplescreen results and hyperechoic fetal bowel. Am 7 Obstet Gynaecol 1995;953-4

3 Lynch L, Ghidni A. Perinatal infections (Review). Curr Opin Obstet Gynaecol 1993;5:24-32.

4 Raynor BD. Cytomegalovirus infection in pregnancy (Review). Semin Perinatol 1993;17:394-402.
5 de Sarcus B. Cytomegalovirus infections during pregnancy. Presse Med 1996;25:211-2.

6 Elder SP. Current prospects for immunisation against cytomegalovirus disease (Review). Infect Agents Dis 1996;5: 29-35.

7 Hudecz F, Gonczol E, Poltkin SA. Preparation of highly purified human cytomegalovirus envelope antigen vaccine. Vaccine 1985;3:300-4. 